University of Nebraska - Lincoln

DigitalCommons@University of Nebraska - Lincoln

US Army Research

U.S. Department of Defense

2011

Protective immunity to pre-erythrocytic stage malaria

Robert J. Schwenk

Walter Reed Army Institute of Research

Thomas L. Richie

Naval Medical Research Center, Silver Springs, MD

Follow this and additional works at: https://digitalcommons.unl.edu/usarmyresearch

Part of the Operations Research, Systems Engineering and Industrial Engineering Commons

Schwenk, Robert J. and Richie, Thomas L., "Protective immunity to pre-erythrocytic stage malaria" (2011). US Army Research. 105.

https://digitalcommons.unl.edu/usarmyresearch/105

This Article is brought to you for free and open access by the U.S. Department of Defense at DigitalCommons@University of Nebraska - Lincoln. It has been accepted for inclusion in US Army Research by an authorized administrator of DigitalCommons@University of Nebraska - Lincoln. 


\title{
Protective immunity to pre-erythrocytic stage malaria
}

\author{
Robert J. Schwenk ${ }^{1,2}$ and Thomas L. Richie ${ }^{1,3}$ \\ ${ }^{1}$ US Military Malaria Vaccine Program \\ ${ }^{2}$ Walter Reed Army Institute of Research, Division of Malaria Vaccine Development, 503 Robert Grant Avenue, Silver Spring, \\ MD 20910, USA \\ ${ }^{3}$ Naval Medical Research Center, Infectious Disease Directorate, 503 Robert Grant Avenue, Silver Spring, MD 90910, USA
}

\begin{abstract}
The development of a vaccine against malaria is a major research priority given the burden of disease, death and economic loss inflicted upon the tropical world by this parasite. Despite decades of effort, however, a vaccine remains elusive. The best candidate is a subunit vaccine termed RTS,S but this provides only partial protection against clinical disease. This review examines what is known about protective immunity against pre-erythrocytic stage malaria by considering the humoral and $T$ cell-mediated immune responses that are induced by attenuated sporozoites and by the RTS,S vaccine. On the basis of these observations a set of research priorities are defined that are crucial for the development of a vaccine capable of inducing long-lasting and high-grade protection against malaria.
\end{abstract}

\section{The scope of the problem}

Malaria is caused by the protozoan genus Plasmodium and is responsible for 700000 to 1000000 deaths per year in tropical regions of the world, most severely affecting children in sub-Saharan Africa [1]. Naturally acquired immunity to malaria is slow to develop, non-sterilizing and short-lived in the absence of continuous exposure. The parasite rapidly develops resistance to anti-malarial drugs and treatment is often followed by reinfection [2]. Hence, there is an urgent need to develop a malaria vaccine.

This task has proven difficult: after decades of effort, the only candidate vaccine approaching licensure is RTS,S [3], a virus-like particle, based on the dominant surface protein [designated the circumsporozoite protein (CS protein)] of the Plasmodium falciparum ( $P f$ ) sporozoite. RTS,S has the potential to provide a significant health benefit if the reductions in clinical illness seen in early field trials are confirmed in the Phase III trial currently underway in nine countries across sub-Saharan Africa [3]. However, RTS,S does not appear potent enough to prevent infection completely in the majority of vaccine recipients, inducing only partial immunity. To design a vaccine providing lifelong, sterile protection, it will be important to understand better the interface of the parasite with the human immune system, including its immune-evasion mechanisms. This objective is especially relevant for the early (preerythrocytic) sporozoite and liver stages of the infection where the induction of sterilizing immunity would

Corresponding author: Schwenk, R.J. (robert.schwenk@amedd.army.mil). completely prevent blood stage malaria and thus both clinical illness and transmission.

This review highlights what is known about the immunological responses to the pre-erythrocytic stages of the parasite, focusing on two models of protection: immunization with attenuated sporozoites and immunization with the CS protein. It then identifies key research questions to guide the development of a highly effective pre-erythrocytic stage vaccine.

\section{The sporozoite}

When an infected female Anopheles mosquito bites, it injects several hundred sporozoites into the cutaneous tissue. Over the next minutes to hours some of the sporozoites leave the injection site and enter blood vessels where they are carried rapidly to the liver, while others remain in the skin or travel to regional lymph nodes or to the spleen. On reaching the liver, sporozoites cross the endothelium, in some cases passing through Kupffer cells $(\mathrm{KC})$, and then traverse several hepatocytes before entering a terminal hepatocyte via an invagination process, leading to the formation of a parasitophorous vacuole in which each sporozoite differentiates into liver stage and finally blood stage parasites [4-6] (Figure 1). As the sporozoite passes through a hepatocyte it can cause cell wounding [4] and in some instances necrosis, although whether or not this constitutes a danger signal for the immune system is unclear. The liver stage of infection appears otherwise to be relatively immunologically silent, possibly due to the minor antigen (Ag) load, the immunetolerant nature of the liver, and/or the immune-regulatory mechanisms initiated during sporozoite passage through the skin [7].

\section{The attenuated sporozoite human protection model} In 1973 it was demonstrated that humans subjected to approximately 1000 to 2000 irradiated and $P f$ sporozoiteinfected mosquito bites, followed by primary challenge within 10 weeks after immunization, were protected and the protection lasted for up to 10 months against sporozoite re-challenge $[8,9]$. This work followed studies where immunization of animals with radiation-attenuated sporozoites was similarly highly protective $[10,11]$. Recently, immunization of rodents with genetically attenuated sporozoites (GAS) also afforded high-grade $(>90 \%)$ protection [12]. Given the powerful immunity induced by attenuated 


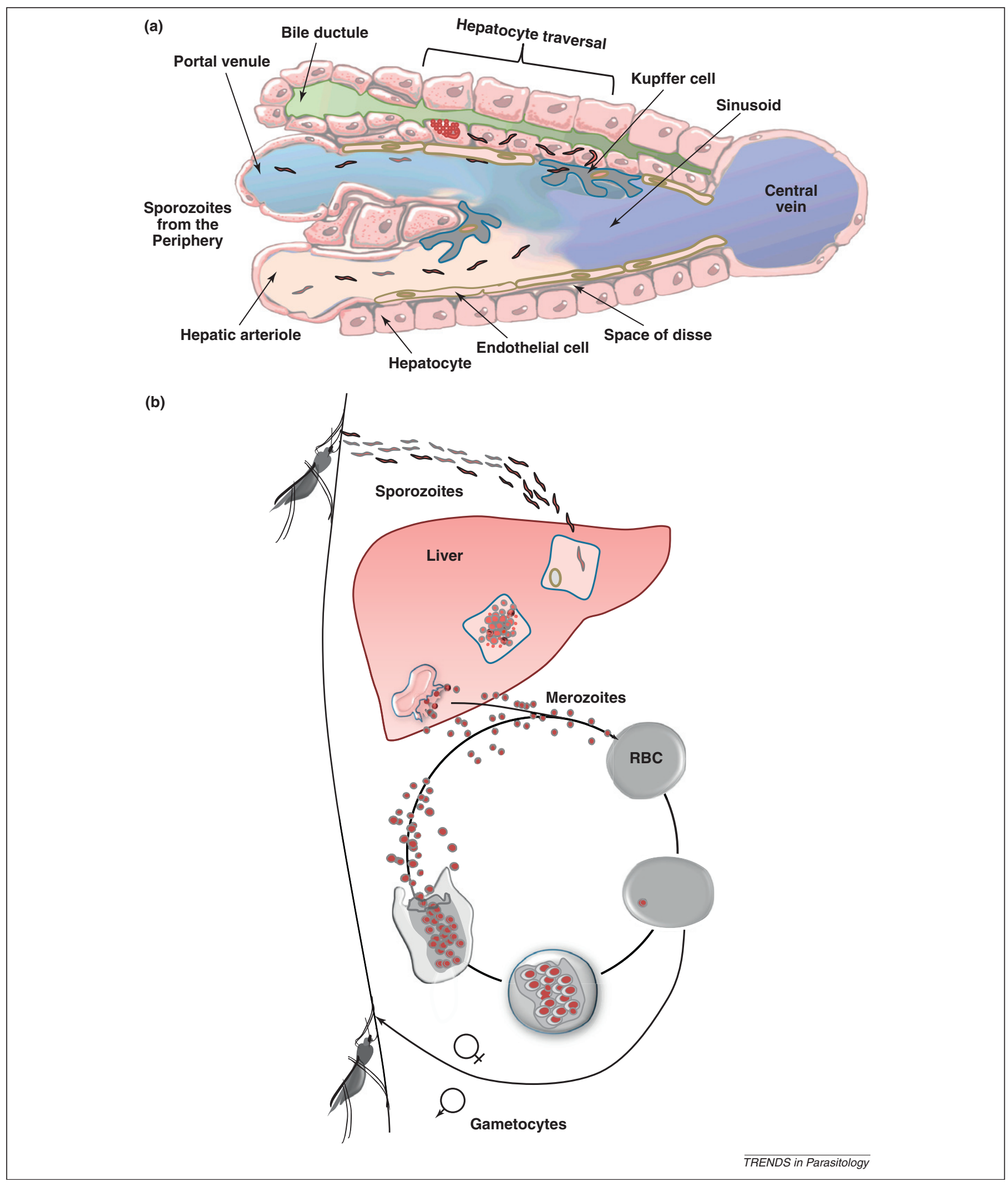

Figure 1. The Plasmodium life cycle. (a) Sporozoites enter the liver lobule either via the portal venule or the hepatic artery, and arrest by binding to the sinusoidal cell layer. The parasites glide along the sinusoid, sometimes moving against the flow of blood, until they encounter a Kupffer cell. After a pause, they slowly pass through the Kupffer cell and cross the space of Disse beyond it. Once inside the liver parenchyma, the parasites increase their velocity and migrate for many minutes through several hepatocytes, before they eventually come to rest in a terminal hepatocyte to initiate development (panel reproduced with permission from [5]). (b) An infected female Anopheles injects up to several hundred sporozoites into the cutaneous tissue while feeding. Some of the sporozoites leave the injection site and enter blood vessels where they are carried to the liver. Some of the sporozoites invade hepatocytes (panel (a)) where they undergo asexual multiplication (schizogony) and develop into thousands of merozoites. The merozoites exit the infected hepatocyte in membrane-bound vesicles (merosomes or extrusomes) which, following rupture, enable the merozoites to rapidly invade red blood cells thereby initiating blood-stage malaria. During the blood stage, formation of gametocytes also occurs and these are ingested by a feeding mosquito to initiate the next cycle. 
sporozoites, much effort has been expended to identify the underlying protective mechanisms, including the antigenic targets.

Radiation-attenuated sporozoites (RAS), as do native sporozoites, invade hepatocytes and form a parasitophorous vacuole, but their development is arrested at the early liver stages [13], creating a repository of early liver stage Ags which could serve to induce and maintain protracted immunity. The relatively persistent Ag load is one factor that distinguishes immunization with attenuated sporozoites from exposure to intact sporozoites under natural conditions. The CS protein and probably other parasite Ags cross the vacuolar membrane, possibly via a 'gatekeeper complex' [14], to enter the cytoplasm where they undergo processing and subsequent presentation on the hepatocyte surface. It has been demonstrated that sporozoite-infected hepatocytes can process and present Plasmodium berghei $\mathrm{Pb}$ ) CS-protein epitopes to T cells [15], although it is also possible that sporozoites and liver-stage Ags egress from the cell to be ingested and processed by dendritic and other Ag-presenting cells. Likewise, some of the RAS entering the liver are directly taken up by dendritic cells (DC) for processing and presentation to $\mathrm{T}$ cells. It is probable that multiple antigens are involved in protection, in as much as CS protein contributes to but is not required for protection, because mice tolerized to CS protein can still be protected by RAS [16].

\section{The RTS,S human protection model}

The surface of the sporozoite is densely packed with protein. The most prominent is the CS protein which is shed as the parasite undergoes motility [17] and cell traversal [18]. The $P f$ CS protein has $\mathrm{N}$ and $\mathrm{C}$ terminal regions which flank a central repeat region consisting of 38 to 40 copies of the B cell epitope NANP, preceded by a minor central repeat sequence of alternating NVDP and NANP. Several CD4 and CD8 T cell epitopes have been mapped to the C terminus of the CS protein, and some of these are universal epitopes [19-22]. Currently, there are also extensive efforts to identify additional sporozoite proteins [23-25].

The relatively potent immunogenicity and prominent location on the sporozoite surface have made the CS protein a leading candidate $\mathrm{Ag}$ for malaria vaccines. Following the demonstration that CS-protein-based vaccines can induce protection in animals, the RTS,S vaccine was developed to extend this protection to humans [3,26]. RTS,S consists of the central repeat region and the $\mathrm{C}$ terminus of the $P f$ CS protein fused to the surface $\mathrm{Ag}$ of hepatitis $\mathrm{B}$ virus; this is coexpressed with free surface $\mathrm{Ag}$ to form an hepatitis B surface (HBs) Ag-like particle. RTS,S has been formulated in different adjuvants including ASO2A, an oilin-water emulsion containing QS-21 (a derivative of the saponin Quil A) and mono-phosphoryl lipid A, and ASO1B, which is a liposome formulation. The RTS,S vaccine confers sterile immunity to $40-50 \%$ of malaria-naïve subjects against a primary sporozoite challenge, and $40-50 \%$ of subjects protected against the primary challenge are also protected against rechallenge 5 months later [27]. The Aband cell-mediated immune responses to RTS,S have been characterized extensively in both protected and non-protected human volunteers $[27,28]$.

\section{Ab-mediated immunity}

There is evidence that humoral immunity contributes to protection against pre-erythrocytic stage malaria. Passive transfer of monoclonal $\mathrm{Ab}$ specific for the central repeat regions of $P b$ and $P$. yoelii $(P y) C S$ protein protects mice against sporozoite challenge $[29,30]$. Immune serum from sporozoite-immunized humans can also block the invasion of $P f$ sporozoites into hepatocytes [31]. In addition, the RTS,S vaccine induces high titers of CS-protein-specific Abs that correlate with protection against infectious challenge. The mechanism underlying Ab-mediated protection could involve inhibition of hepatocyte invasion or opsonization of sporozoites for uptake by macrophages and DC. Recent studies [32] have also shown that sporozoites injected into the dermis of immunized mice are immobilized and become degraded within hours. Moreover, mosquitoes introduce fewer sporozoites into immunized as compared to non-immunized mice, presumably due to the formation of $\mathrm{Ag}-\mathrm{Ab}$ complexes in the proboscis [32]. Consequently, RTS,S-induced Abs probably also inhibit sporozoite motility.

Because sporozoites invade hepatocytes soon after injection, sufficiently high titers of sporozoite-specific Abs must be present at the time of injection for Abs to confer protection. This implies a need for sustained Ab production by long-lived plasma cells (PC) (Figure 2). The activation of these cells appears to involve Ags expressing both a repetitive epitope to mediate high $\mathrm{B}$ cell receptor cross-linking and $\mathrm{T}$ cell epitopes to induce the activation of follicular Thelper $\left(\mathrm{T}_{\mathrm{FH}}\right)$ cells [33]. Particular attenuated virus vaccines meet these criteria and can induce $\mathrm{Ab}$ responses that persist for decades without apparent re-boosting [34,35]. Long-term storage of $\mathrm{Ag}-\mathrm{Ab}$-complement complexes on follicular DC [36] could also be required.

For the RTS,S vaccine the majority of non-protected volunteers make much weaker CS-protein-specific $\mathrm{Ab}$ responses than protected volunteers. In addition, CS-protein-specific $\mathrm{Ab}$ titers fall substantially with time after the $3^{\text {rd }}$ dose, even when accompanied by natural boosting [37]. Hence, mechanisms that sustain high Ab titers would probably also improve long-term vaccine efficacy. In general, higher primary $\mathrm{Ab}$ titers and longer intervals between boosting tend to favor longer-lived $\mathrm{Ab}$ responses, and it would therefore be worth evaluating at 0,1 , and 6 months instead of in the standard 0,1 , and 2 months immunization schedule. There is also evidence [38] that high avidity Abs are more protective than low-avidity Abs, thus methods to increase $\mathrm{Ab}$ avidity would probably improve RTS,S-induced protection.

\section{CD4 $\mathrm{T}$ cell immunity}

There is evidence that $\mathrm{CD} 4 \mathrm{~T}$ cells also protect against the pre-erythrocytic stages of malaria. CD4 T cells specific for a $P y$ CS-protein epitope can both eliminate infected hepatocytes in vitro and adoptively transfer protection in vivo [39]. As mentioned, the RTS,S vaccine induces high titers of CS-protein-specific IgG Abs, and this probably depends on the activation of CS-protein-specific CD4 $\mathrm{T}_{\mathrm{FH}}$ cells. RTS,S-primed CD4 T cells can also be recalled with CSprotein peptides to produce IFN- $\gamma$ [27], a known inhibitor of intracellular stages of the parasite [40]. Moreover, the 


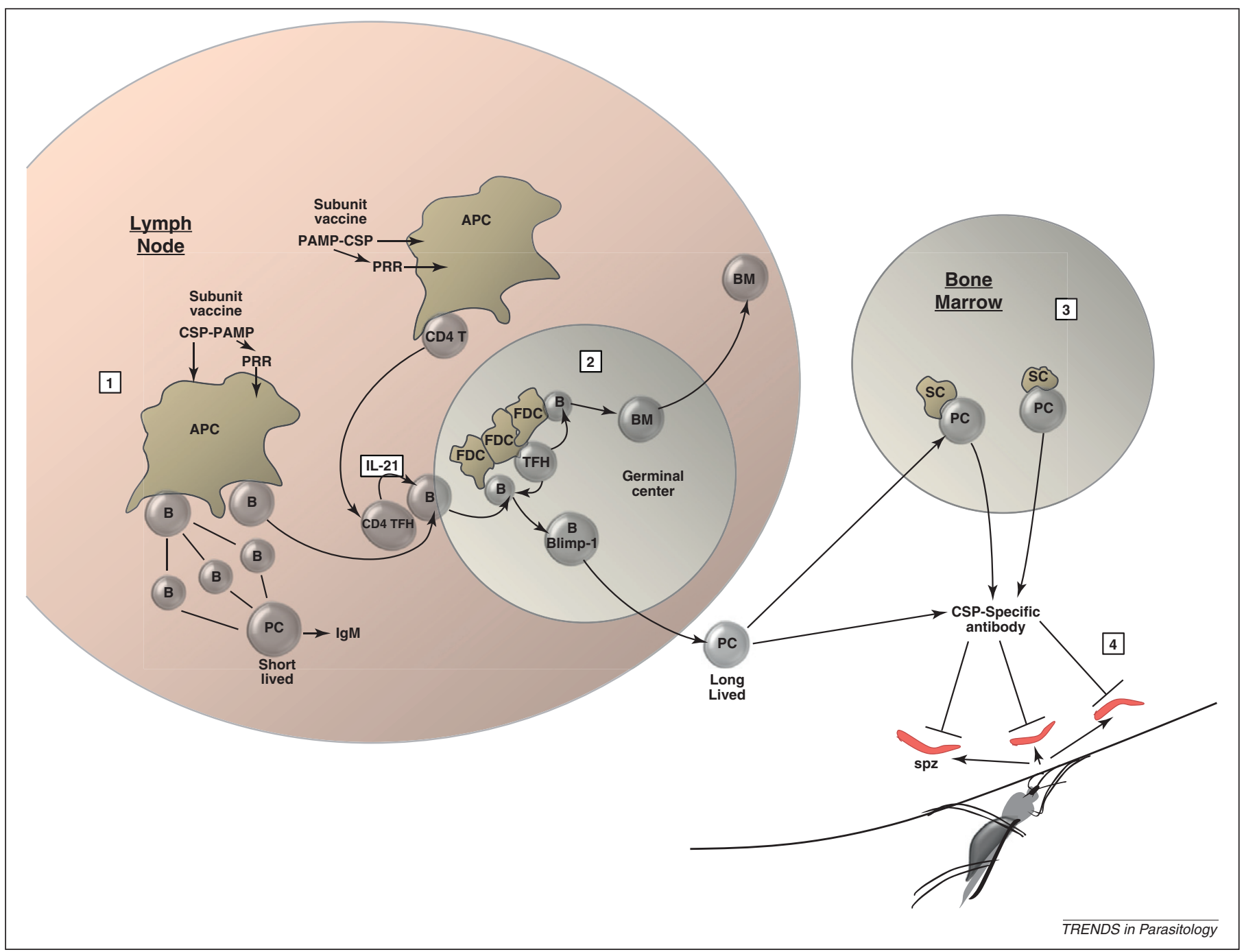

Figure 2. Induction of humoral immunity. Region 1: a subunit vaccine - in this case the circumsporozoite (CS) protein coupled to a pathogen-associated molecular pattern

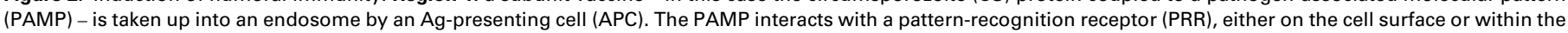
endosome, and this stimulates maturation of the APC (Figure 3 legend). Following digestion of the protein within the endosome and binding of the resulting peptide fragments to the binding-grooves of class II major histocompatibility molecules, recognition of CS-protein peptide plus class II molecule on the APC surface by an Agspecific $C D 4^{+} T$ cell induces upregulation of $C X C R 5$ and differentiation of the $C D 4^{+} T$ cell into a follicular $T$-helper cell $\left(T_{F H}\right)$. B cell activation also occurs, causing some $B$ cells

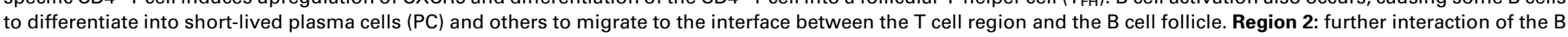

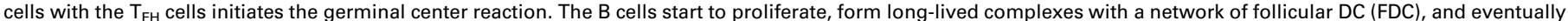
differentiate into memory $B\left(B_{M}\right)$ cells or into B-lymphocyte-induced maturation protein (Blimp-1) positive B cells and then into long-lived PC. Region 3: the PCs migrate to

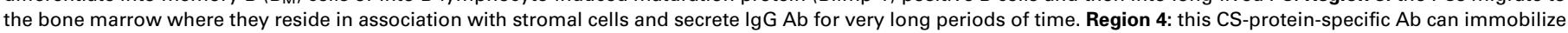
sporozoites immediately following injection by the mosquito and hence can confer protection against infection.

frequency of the RTS,S-induced IFN- $\gamma^{+}$CD4 T cells was higher in protected than in non-protected volunteers [27].

Studies of other pathogens in both mice [41] and humans [42] indicate that multifunctional CD4 T cells simultaneously producing IL-2, IFN- $\gamma$ and TNF- $\alpha$ more strongly associate with protection than bi- or mono-functional CD4 T cells. This could be due to the capacity of IL-2 to promote lymphoproliferation and hence maintain the lineage and the capacity of IFN- $\gamma$ and TNF- $\alpha$ to act synergistically. In addition, the intensity of IFN- $\gamma$ secretion from multifunctional cells appears to be greater than that of terminal effector cells. Seder et al. [43] have proposed a model in which CD4 $\mathrm{T}$ cells progressively gain and then eventually lose effector function as they proceed along the following linear differentiation pathway: IL- $2^{+} \mathrm{TNF}-\alpha^{+}$ cells $\rightarrow$ IL- $2^{+}$TNF- $\alpha^{+}$IFN- $\gamma^{+}$cells $\rightarrow\left[\right.$ TNF- $\alpha^{+}$IFN- $\left.\gamma^{+}\right]$or $\left[\mathrm{IL}-2^{+}\right.$IFN- $\left.\gamma^{+}\right]$cells $\rightarrow$ IFN- $\gamma^{+}$-only terminal effector cells.
They propose that an ideal vaccine will be strong enough to drive CD4 T cells to differentiate into IL- $2^{+}$TNF- $\alpha^{+}$IFN- $\gamma^{+}$ triple producers (referred to as the 'sweet spot'), but not so strong as to further differentiate them into terminal effectors.

The CD4 $\mathrm{T}$ cell lineage also includes regulatory $\mathrm{T}\left(\mathrm{T}_{\text {reg }}\right)$ cells which are generally controlled by the transcription factor FOXP3 (reviewed in [44]) and which limit excessive inflammation and inhibit sterilizing immunity to pathogens. For example, $\mathrm{T}_{\text {reg }}$ cells prevent effector $\mathrm{T}$ cells from completely eliminating Leishmania parasites from the site of infection, and the persistence of residual parasites contributes to the maintenance of long-term immunity [45]. There are several reports that $\mathrm{T}_{\text {reg }}$ cells control immune responses to blood stage malaria [46,47] and, although there is no direct evidence, they could also regulate responses to the pre-erythrocytic stages. It is clear that 
future immunization strategies might have to consider whether a vaccine activates $T_{\text {reg }}$ cells in addition to effector and memory $\mathrm{T}$ cells and, if this is the case, identify a method to counteract the effects of $\mathrm{T}_{\text {reg }}$ cells such as the coadministration of an immunomodulatory drug [46].

\section{CD8 T cell immunity}

Although Abs and CD4 T cells contribute to protection, CD8 T cells appear to be the sine qua non effectors that confer sterile immunity in some strains of attenuated sporozoite-immunized mice. Thus, for example, depletion of $\mathrm{CD}^{+}$cells from RAS-immunized BALB/c mice before challenge abrogates protection. In addition, CD8 transgenic T cells specific for Py CS protein can secrete IFN- $\gamma$ in response to and protect mice against sporozoite challenge. Recently it has been demonstrated that GAS immunization of mice also activates CD8 T cells that are required for protection [48] and which can effect contact-dependent killing of $P y$-infected hepatocytes [49].

CD8 T cells specific for Pf CS protein have also been found in the blood of both RAS-immunized volunteers and naturally exposed subjects $[21,50]$. In addition, investigators working at the University of Oxford have explored the use of vaccines encoding the pre-erythrocytic stage thrombospondin-related adhesion protein Ag (TRAP) fused to a string of linked T- and B-cell epitopes (ME-TRAP), and showed that priming humans with a construct of chimpanzee $(\mathrm{Ch})$ adenovirus 63 encoding ME-TRAP (AdCh63-METRAP), followed by a boost with a modified vaccinia Ankara (MVA) construct MVA-ME-TRAP, induced strong CD8 $\gamma$-interferon-secreting $\mathrm{T}$ cell responses and the associated efficacy data has been submitted for publication (Ewer et al., submitted, Adrian Hill, personal communication).

There is evidence that some sporozoites migrate from the dermis to the draining lymph node where they activate CS-protein-specific CD8 T cells [51]. These CD8 T cells subsequently travel to the liver where they could contribute to pre-erythrocytic stage immunity. It is more likely, however, that attenuated sporozoites also induce CD8 T cells that are specific for the liver-stage Ags developing only in infected hepatocytes.

Attenuated sporozoites activate CD8 T cells, whereas native sporozoites do not, and this could be related to the former causing infected hepatocytes to undergo apoptosis $[52,53]$. The apoptotic cells are probably taken up by intrahepatic $\mathrm{CD}^{+} \mathrm{DC}$ for cross-presentation of liver-stage Ags to CD8 T cells [54]. This mechanism, however, has been called into question [55], in part because native sporozoites can also induce sterile immunity in humans when administered with chloroquine to prevent blood stage infection [56], although there is the additional proviso that immunity in this case could have been directed primarily against very early blood stages that exit the liver. Protection, in this case, appears to be mediated by multi-functional effector memory $\mathrm{T}\left(\mathrm{T}_{\mathrm{EM}}\right)$ cells, and chloroquine could have potentiated the activation of these cells [57].

In general, acute infection of humans or mice with a pathogen causes CD8 T cells to undergo rapid expansion to generate a large population of effector cells. Upon pathogen clearance, the cells undergo rapid contraction when most of the cells die by apoptosis. However, 5-10\% of the effector cells survive and form long-lived memory CD8 T cells. The extent of contraction, and the maintenance of memory cells, appear to depend on several $\gamma_{c}$ cytokines including IL-7, IL-15 and IL-21. Immunization of mice with RAS or GAS results in the accumulation in the liver of both $\mathrm{CD} 44^{+} \mathrm{CD}^{-} \mathrm{L}^{-} \mathrm{CD} 122^{-} \mathrm{CD}^{+}$effector/ effector memory $\mathrm{T}\left(\mathrm{T}_{\mathrm{E} / \mathrm{EM}}\right)$ cells and $\mathrm{CD} 44^{+} \mathrm{CD} \mathrm{L}^{+}$ $\mathrm{CD}_{122^{+}} \mathrm{CD}^{+}$central memory $\mathrm{T}\left(\mathrm{T}_{\mathrm{CM}}\right)$ cells $[58,59]$. Surprisingly, the CD8 $\mathrm{T}_{\mathrm{E} / \mathrm{EM}}$ cells do not undergo contraction after the last boost/immunization or after sporozoite challenge, even after a prolonged period of time. It is conceivable that the lack of contraction is due to chronic Ag stimulation, and this would be consistent with RAS immunization generating a persisting Ag depot. It is not clear whether protection against sporozoite challenge requires the protracted presence of $\mathrm{CD} 8 \mathrm{~T}_{\mathrm{E}}$ cells that could respond immediately to infection. If, by contrast, protection depends on CD8 $\mathrm{T}_{\mathrm{CM}}$ cells that can only be recruited from a draining lymph node, there could be insufficient time for these cells to recognize new parasite $\mathrm{Ag}$, multiply to high numbers, and migrate to the liver to kill the parasite. Conversely, if CD8 $\mathrm{T}_{\mathrm{CM}}$ cells are maintained in the liver, temporal constraints might not apply. One of the key questions for the development of a malaria subunit vaccine is, therefore, whether protection against sporozoite challenge requires persisting CD8 $\mathrm{T}_{\mathrm{E}}$ cells or CD8 $\mathrm{T}_{\mathrm{CM}}$ cells and, if the former, whether the maintenance of these cells depends on a persisting depot of Ag. It is worth noting that primaquine treatment to eliminate persisting liver-stage Ag before the development of longlived memory cells abolished protection in both RAS [60] and GAS [61]-immunized animals.

Valuable data regarding the role of central versus effector memory CD8 T cells have been provided by investigators at the University of Oxford. Volunteers were immunized with ME-TRAP; DNA and fowlpox constructs were used for priming, and an MVA construct provided the boost. In some cases, vaccine-induced reduction in liverstage burden correlated more closely with long-term (cells cultured for $9 \mathrm{~d}$ ) than with short-term (cells cultured for $18 \mathrm{~h})$ responses as measured by enzyme-linked immunospot (ELISPOT) assay, indicating that $\mathrm{T}_{\mathrm{CM}}$ cells present at the time of sporozoite challenge could make a greater contribution to protection than $\mathrm{T}_{\mathrm{EM}}$ cells $[62,63]$. A principal hindrance to answering these questions in the RAS model is our lack of knowledge of the Ags expressed by the liver stage of the parasite. Consequently, extensive efforts employing both genomics and proteomics are being directed to discovering relevant liver stage Ags [23-25]. For example, 16 novel $P f$ proteins have been recently identified which peripheral blood mononuclear cells (PBMC) from RAS-immunized volunteers recognized more frequently than 'traditional' Pf Ags such as the CS protein and exported protein-1 (EXP-1) [64]. Moreover, two of these 16 Ags elicited higher responses from protected versus non-protected volunteers.

Once the mechanisms that enable attenuated sporozoites to induce sterile CD8 T-cell-mediated immunity are understood, attempts could be made to trigger similar 
immune mechanisms by a subunit vaccine. The initiation of the appropriate activation pathways will probably occur in the lymph node draining the vaccination site. Figure 3 illustrates the cellular interactions that lead to the activa- tion of short-lived effector CD8 T cells (SLECs), memory potential effector CD8 T cells (MPECs) and memory CD8 T cells, as well as the migration of these cells from the lymph node to the liver to mediate their effector function.

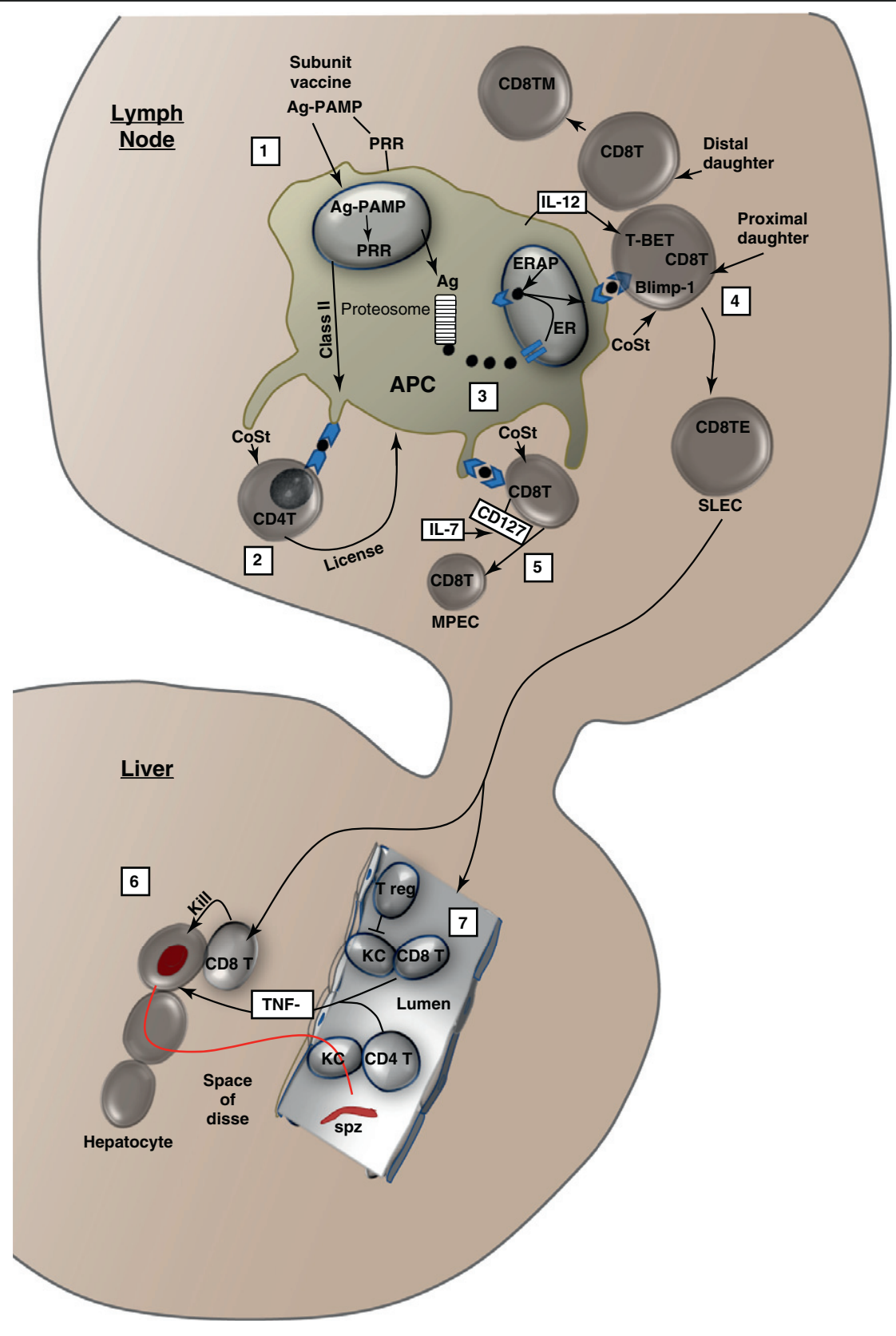

TRENDS in Parasitology

Figure 3. Induction of T cell mediated immunity. Region 1: a subunit vaccine - in this case a liver-stage antigen (Ag) coupled to a pathogen-associated molecular pattern (PAMP) - is taken up into an endosome by an Ag-presenting cell (APC). The PAMP interacts with a pattern-recognition receptor (PRR), either on the cell surface or within the endosome, and this stimulates the maturation of the APC. Region 2: Ag within early endosomes is degraded and binds to nascent class II molecules for transport to the cell

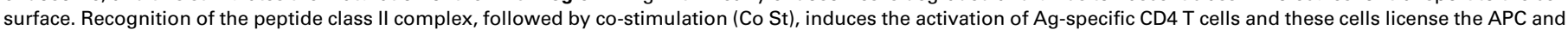
enable it to activate CD8 T cells. Region 3: some Ag is translocated out of the endosome into the cytoplasm where it is degraded into peptides by the proteosome. The peptides are transported into the endoplasmic reticulum (ER) by the transporter associated with antigen processing (TAP) where they are bound to nascent class I molecules and then trimmed to size by the ER aminopeptidase (ERAP) enzymes. The peptide class I complexes are then transported to the cell surface. Region 4: the peptide class I complexes are recognized by Ag-specific $\mathrm{CD}^{+} \mathrm{T}$ cells which undergo proliferation under the influence of costimulation and APC-derived IL-12. The proximal daughter upregulates the T box expressed in T cells transcription factor (T-BET) and Blimp-1 and develops into a short-lived effector cell (SLEC) while the distal daughter becomes a long-lived memory CD8 $T\left(T_{M}\right)$ cell. Region 5: during CD8 T cell expansion some effector cells do not down regulate CD127, and under the influence of IL-7 differentiate into memory potential effector cells (MPECS). Region 6: the sporozoite migrates through the Kupffer cell (KC) and several hepatocytes to lodge in a terminal hepatocyte within a parasitophorous vacuole. The CD8 effector T cells leave the lymph node and migrate to the space of Disse or into the sinusoid of the liver. They recognize parasite Ag plus class I on the surface of the infected hepatocyte and elaborate IFN- $\gamma$ and perforin/granzyme (P/G) to kill the parasite. Region 7: as a result of cell traversal or of protein shedding, KC and other APC also process and present parasite Ags to activate CD4 and CD8 T cells in the sinusoid of the liver. These cells produce $\mathrm{TNF}-\alpha$ which can also act at a distance to kill the parasite. The cytokine milieu of the liver might also promote the activation of regulatory $\mathrm{T}$ cells $\left(\mathrm{T}_{\text {reg }}\right.$ ), and these could secrete IL-10 to suppress the activation of effector T cells. 


\section{Key research questions}

RTS,S vaccine trials have shown that both Abs and CD4 T cells specific for the CS protein confer sterile immunity to about $50 \%$ of malaria-naïve volunteers and partial immunity to children in endemic areas. RAS sporozoite vaccines also confer sterile immunity and, in the mouse model, CD8 $\mathrm{T}$ cells specific for currently unidentified liver stage Ags appear to be the key mediators of protection. Most recently, data from a fowlpox prime and MVA boost regimen (using the ME-TRAP antigen) suggest that $\mathrm{T}$ lymphocytes can provide sterile immunity in humans in the absence of significant antibody responses [65]. Hence, there appear to be multiple immune responses that can be harnessed to achieve pre-erythrocytic stage immunity. Key outstanding questions regarding the induction and maintenance of protective immunity to pre-erythrocytic stage antigens are listed in Table 1.

\section{Conclusions and future directions}

Long-term maintenance of high titers of sporozoite surfaceAg-specific Abs will be an important component of the protective immunity induced by vaccines, probably depending upon the generation of long-lived PCs. In general, stronger primary Ab responses correlate with stronger and more long-lived booster responses, and therefore adjuvants and vaccine platforms that favor strong primary responses could contribute to the longevity of the $\mathrm{Ab}$ response. Longer times between prime and boost immunizations also favor long-lived Ab production [66]. Enhancing the expression of the transcription repressor Blimp-1, which promotes the differentiation of $\mathrm{B}$ cells into long-lived PC [67], might be achieved by activating IL-21-secreting $\mathrm{T}_{\mathrm{FH}}$ cells, and this could also favor durable $\mathrm{Ab}$ production.

An effective malaria vaccine should also induce strong and long-lasting pre-erythrocytic stage $\mathrm{T}$ cell immunity.
The identification of the most protective pre-erythrocytic stage Ags is a primary objective. It will be necessary to establish whether intra-hepatic $\mathrm{T}_{\mathrm{E}}$ cells or $\mathrm{T}_{\mathrm{CM}}$ cells are the main contributors to protracted $\mathrm{T}$ cell immunity and to confirm whether the preservation of $\mathrm{T}_{\mathrm{E}}$ cells requires a persistent Ag depot. It follows that adjuvants that create an $\mathrm{Ag}$ depot or a recombinant virus generating persistent latent infection [68] might help to maintain multi-functional $\mathrm{T}_{\mathrm{EM}}$ cells in the peripheral tissue. The highly successful yellow fever vaccine targets four different TLRs located on distinct sub-populations of DCs, each with its own unique booster-effect on the immune system [69]. Hence, a combination of malaria Ags with multiple component adjuvants that target different TLRs could lead to a $\mathrm{T}_{\mathrm{H} 1} / \mathrm{T}_{\mathrm{H} 2}$ balance favoring long-lived $\mathrm{Ab}$ production and long-lived $\mathrm{T}$ cell memory. Other adjuvants such as Iscomatrix (cage-like structures consisting of saponin, phospholipid and cholesterol) could also be considered, to enhance $\mathrm{Ab}$ as well as CD4 and CD8 T cell responses in humans [70].

Alternatively, vaccine platforms with intrinsic immunostimulatory properties could be employed to achieve a similar broad activation of humoral and cellular immunity. Combining RTS,S with a viral vector expressing either CS protein or other appropriate sporozoite or liver-stage Ags in a prime and boost regimen could induce both strong $\mathrm{Ab}$ and CD4 and CD8 T cell responses leading to more durable protection [71]. In support, a study in macaques demonstrated that two doses of RTS,S following an adenovirus 35 CS-protein priming immunization retained the RTS,S-induced $\mathrm{Ab}$ response while promoting a markedly higher CD4 T-cell response that remained elevated for about 2 months [72].

Finally, a subunit vaccine will need to be immunogenic in humans, who generally exhibit a high degree of genetic

Table 1. Outstanding issues in malaria vaccine development

\begin{tabular}{|c|c|}
\hline Ab-mediated immunity & Refs \\
\hline Identify mechanisms that maintain high titers of CS-protein-specific Abs for a prolonged period of time & {$[33-36,66,67,69]$} \\
\hline $\begin{array}{l}\text { Establish the contribution to protection of Ab responses targeting malaria proteins expressed on the } \\
\text { surface of infected hepatocytes }\end{array}$ & [75] \\
\hline Identify mechanisms to induce production of high-avidity Abs & [38] \\
\hline \multicolumn{2}{|l|}{ T cell-mediated immunity } \\
\hline Identify the key sporozoite and liver-stage Ags that stimulate protective CD4 and CD8 T cell-mediated immunity & {$[23-25,64]$} \\
\hline $\begin{array}{l}\text { Establish whether there is a need for chronic stimulation from a persisting } \mathrm{Ag} \text { depot to maintain long-lived } \\
\text { intra-hepatic CD4/CD8 } T_{E} \text { cells }\end{array}$ & {$[36,49,60,61]$} \\
\hline $\begin{array}{l}\text { Characterize the relative contribution of multi-functional CD4/CD8 T cells versus unifunctional CD4/CD8 } \\
T \text { cells in conferring protection }\end{array}$ & {$[27,41,42]$} \\
\hline $\begin{array}{l}\text { Identify the primary site (liver or lymph node draining the liver) of pre-erythrocytic stage Ag-specific } \\
\text { CD4/CD8 T cell activation }\end{array}$ & {$[15,51,54,69]$} \\
\hline $\begin{array}{l}\text { Establish whether } \mathrm{CD} 4 \mathrm{~T}_{\mathrm{FH}} \text { cells promote the differentiation of pre-erythrocytic stage-specific } \mathrm{B}_{\mathrm{M}} \\
\text { cells and long-lived } \mathrm{PC} \text { cells }\end{array}$ & [33] \\
\hline Determine whether regulatory T cells inhibit responses to pre-erythrocytic stage Ags & {$[7,44-47,56]$} \\
\hline $\begin{array}{l}\text { Elucidate to what extent host and parasite genetic diversity affect CD4/CD8 T-cell responses to } \\
\text { pre-erythrocytic stage Ags }\end{array}$ & {$[19,21,79]$} \\
\hline
\end{tabular}


diversity. One approach to overcoming genetic restriction would be to employ a subunit vaccine containing epitopes from multiple antigens, including universal epitopes that bind to multiple HLA types. In addition, it might be possible to choose epitopes that are restricted by HLA supertypes.

In summary, a successful pre-erythrocytic stage malaria vaccine will have to induce a durable antibody response against the sporozoite and a protracted CD4 and CD8 protective $\mathrm{T}$ cell response against the liver stages of the parasite. These goals should be achievable using the appropriate immunization regimens in conjunction with newly developed adjuvants and vaccine delivery vehicles. A malaria vaccine will also have to address human genetic diversity, possibly by including universal $\mathrm{T}$ cell epitopes, as well as the genetic diversity in the parasite itself. Clearly, we are moving closer to the development of a successful malaria vaccine, but much work remains to be done.

\section{Disclaimer}

The study protocols for the clinical trials discussed in this manuscript that were performed by the US Navy or US Army were approved by the Naval Medical Research Center or Walter Reed Army Institute of Research Institutional Review Boards, respectively, in compliance with all applicable Federal regulations governing the protection of human subjects. The animal experiments reported herein that were conducted by the US Navy or US Army were conducted in compliance with the Animal Welfare Act and in accordance with the principles set forth in the Guide for the Care and Use of Laboratory Animals, Institute of Laboratory Animals Resources, National Research Council, National Academy Press, 1996. T.L.R. is a military service member and R.S. is a contract employee of Clinical Research Management, Hinkley, Ohio. The work of T.L.R. and R.S. was prepared as part of official government duties. Title 17 U.S.C. $\$ 105$ provides that 'Copyright protection under this title is not available for any work of the United States Government.' Title 17 U.S.C. \$101 defines a US Government work as a work prepared by a military service member or employee of the US Government as part of that person's official duties. The work of T.L.R. was supported by work unit number 6000.RAD1.F. A0309.

The views expressed in this article are those of the authors and do not necessarily reflect the official policy or position of the Department of the Navy, the Department of the Army, the Department of Defense, or the US Government.

\section{Acknowledgments}

We gratefully acknowledge Ute Frevert of New York University for allowing us to modify and republish panel (a) of Figure 1.

\section{References}

1 WHO (2009) World Malaria Report, World Health Organization

2 Wells, T. and Poll, E. (2010) When is enough enough? The need for a robust pipeline of high-quality antimalarials. Discov. Med. 9, 389-398

3 Vekemans, J. et al. (2009) Development of the RTS,S/AS malaria candidate vaccine. Vaccine 27 (Suppl. 6), G67-71

4 Mota, M. (2001) Migration of Plasmodium sporozoites through cells before infection. Science 291, 141-144

5 Frevert, U. et al. (2005) Intravital observation of Plasmodium berghei sporozoite infection of the liver. PLoS Biol. 3, 1034-1046
6 Frevert, U. (2004) Sneaking in through the back entrance: the biology of malaria liver stages. Trends Parasitol. 20, 417-424

7 Guilbride, D. et al. (2010) Why functional pre-erythrocytic and bloodstage malaria vaccines fail: a meta-analysis of fully protective immunizations and novel immunological model. PLoS ONE 5, e10685e10706

8 Clyde, D. et al. (1973) Immunization of man against sporozoite-induced falciparum malaria. Am. J. Med. Sci. 266, 169-177

9 Hoffman, S. et al. (2002) Protection of humans against malaria by immunization with radiation-attenuated Plasmodium falciparum sporozoites. J. Infect. Dis. 185, 1155-1164

10 Nussenzweig, R. et al. (1967) Protective immunity produced by the injection of x-irradiated sporozoites of Plasmodium berghei. Nature $216,160-162$

11 Nussenzweig, R. et al. (1972) Sporozoite-induced immunity in mammalian malaria. A review. Am. J. Trop Med. Hyg. 21, 722-728

12 Vaughan, A. et al. (2010) Genetically engineered, attenuated whole-cell vaccine approaches for malaria. Hum. Vaccin. 6, 107-113

13 Zechini, B. et al. (1999) Plasmodium berghei development in irradiated sporozoite-immunized C57BL6 mice. Parasitology 118, 335-338

14 Reiff, S. and Striepen, B. (2009) The gatekeeper revealed. Nature 459, 918-919

15 Bongfen, S. et al. (2007) Plasmodium berghei-infected primary hepatocytes process and present the circumsporozoite protein to specific CD8 T cells in vitro. J. Immunol. 178, 7054-7063

16 Kumar, K. et al. (2006) The circumsporozoite protein is an immunodominant protective antigen in irradiated sporozoites. Nature 444, 937-940

17 Stewart, M. and Vanderberg, J. (1988) Malaria sporozoites leave behind trails of circumsporozoite protein during gliding motility. $J$. Protozool. 35, 389-393

18 Singh, A. et al. (2007) Plasmodium circumsporozoite protein promotes the development of the liver stages of the parasite. Cell 131, 492-504

19 Sinigaglia, F. et al. (1988) A malaria T-cell epitope recognized in association with most mouse and human MHC class II molecules. Nature 336, 778-780

20 Nardin, E. and Nussenzweig, R. (1993) T cell responses to preerythrocytic stages of malaria: role in protection and vaccine development against pre-erythrocytic stages. Annu. Rev. Immunol. $11,687-727$

21 Doolan, D. et al. (1997) Degenerate cytotoxic T cell epitopes from $P$. falciparum restricted by multiple HLA-A and HLA-B supertype alleles. Immunity 7, 97-112

22 Calvo-Calle, J. et al. (2005) Human $\mathrm{CD}^{+}{ }^{+} \mathrm{T}$ cells induced by synthetic peptide malaria vaccine are comparable to cells elicited by attenuated Plasmodium falciparum sporozoites. J. Immunol. 175, 7575-7585

23 Sacci, J., Jr et al. (2005) Transcriptional analysis of in vivo Plasmodium yoelii liver stage gene expression. Mol. Biochem. Parasitol. 142, 177183

24 Tarun, A. et al. (2008) A combined transcriptome and proteome survey of malaria parasite liver stages. Proc. Natl. Acad. Sci. U.S.A. 105, 305310

25 Aguiar, J. et al. (2004) High-throughput generation of $P$. falciparum functional molecules by recombinational cloning. Genome Res. 14, 2076-2082

26 Stoute, J. et al. (1997) A preliminary evaluation of a recombinant circumsporozoite protein vaccine against Plasmodium falciparum malaria. N. Engl. J. Med. 336, 86-91

27 Kester, K. et al. (2009) Randomized, double-blind, phase 2a trial of falciparum malaria vaccines RTS,S/AS01B and RTS,S/AS02A in malaria-naive adults: safety, efficacy, and immunologic associates of protection. J. Infect. Dis. 200, 337-346

28 Moorthy, V. and Ballou, R. (2009) Immunological mechanisms underlying protection mediated by RTS,S: a review of the available data. Malaria J. 8, 312-319

29 Potocnjak, P. et al. (1980) Monovalent fragments (Fab) of monoclonal antibodies to a sporozoite surface antigen $(\mathrm{Pb} 44)$ protect mice against malarial infection. J. Exp. Med. 151, 1504-1513

30 Charoenvit, Y. et al. (1991) Monoclonal, but not polyclonal, antibodies protect against Plasmodium yoelii sporozoites. J. Immunol. 146, 1020 1025

31 Hollingdale, M. et al. (1984) Inhibition of entry of Plasmodium falciparum and $P$. vivax sporozoites into cultured cells; an in vitro assay of protective antibodies. J. Immunol. 132, 909-913 
32 Kebaier, C. et al. (2009) Kinetics of mosquito-injected Plasmodium sporozoites in mice: fewer sporozoites are injected into sporozoitesimmunized mice. PLoS Pathogens 5, e1000399

33 McHeyzer-Williams, L. et al. (2009) Follicular helper T cells as cognate regulators of B cell immunity. Curr. Opin. Immunol. 21, 266-273

34 Hatakeyama, S. et al. (2005) Persisting humoral antiviral immunity within the Japanese population after the discontinuation in 1976 of routine smallpox vaccinations. Clin. Diagn. Lab. Immunol. 12, 520-524

35 Poland, J. et al. (1981) Persistence of neutralizing antibody 30-35 years after immunization with 17D yellow fever vaccine. Bull. World Health Organ. 59, 895-900

36 Tew, J. et al. (1997) Follicular dendritic cells and presentation of antigen and costimulatory signals to B cells. Immunol. Rev. 156, 39-52

37 Alonso, P. et al. (2004) Efficacy of the RTS,S/AS02A vaccine against Plasmodium falciparum infection and disease in young African children: randomised controlled trial. Lancet 364, 1411-1420

38 Feng, J. et al. (2009) Antibody quantity versus quality after influenza vaccination. Vaccine 27, 6358-6362

39 Rénia, L. et al. (1991) In vitro activity of $\mathrm{CD} 4^{+}$and $\mathrm{CD} 8^{+} \mathrm{T}$ lymphocytes from mice immunized with a synthetic malaria peptide. Proc. Natl. Acad. Sci. U.S.A. 88, 7963-7967

40 Ferreira, A. et al. (1986) Inhibition of development of exoerythrocytic forms of malaria parasites by gamma-interferon. Science 232, 881-884

41 Darrah, P. et al. (2007) Multifunctional TH1 cells define a correlate of vaccine-mediated protection against Leishmania major. Nat. Med. 13, 843-850

42 Kannanganat, S. et al. (2007) Multiple-cytokine-producing antiviral $\mathrm{CD} 4 \mathrm{~T}$ cells are functionally superior to single-cytokine-producing cells. J. Virol. 81, 8468-8476

43 Seder, R. et al. (2008) T-cell quality in memory and protection: implications for vaccine design. Nat. Rev. Immunol. 8, 247-258

44 Curotto de Lafaille, M. and Lafaille, J. (2009) Natural and adaptive foxp $3^{+}$regulatory $\mathrm{T}$ cells: more of the same or a division of labor? Immunity 30, 626-635

45 Mendez, S. et al. (2004) Role for $\mathrm{CD}^{+}{ }^{+} \mathrm{CD} 25^{+}$regulatory $\mathrm{T}$ cells in reactivation of persistent leishmaniasis and control of concomitant immunity. J. Exp. Med. 200, 201-210

46 Casares, S. and Richie, T. (2009) Immune evasion by malaria parasites: a challenge for vaccine development. Curr. Opin. Immunol. 21, 321-330

47 Todryk, S. et al. (2008) Correlation of memory T cell responses against TRAP with protection from clinical malaria, and CD4 CD25 high T cells with susceptibility in Kenyans. PLoS ONE 3, e2027-e2039

48 Tarun, A. et al. (2007) Protracted sterile protection with Plasmodium yoelii pre-erythrocytic genetically attenuated parasite malaria vaccines is independent of significant liver-stage persistence and is mediated by $\mathrm{CD}^{+} \mathrm{T}$ cells. J. Infect. Dis. 196, 608-616

49 Trimnell, A. et al. (2009) Genetically attenuated parasite vaccines induce contact-dependent $\mathrm{CD}^{+} \mathrm{T}$ cell killing of Plasmodium yoelii liver stage-infected hepatocytes. J. Immunol. 183, 5870-5878

50 Malik, A. et al. (1991) Human cytotoxic T lymphocytes against the Plasmodium falciparum circumsporozoite protein. Proc. Natl. Acad. Sci. U.S.A. 88, 3300-3304

51 Chakravarty, S. et al. (2007) $\mathrm{CD}^{+} \mathrm{T}$ lymphocytes protective against malaria liver stages are primed in skin-draining lymph nodes. Nat. Med. 13, 1035-1041

52 James, E. (2005) Apoptosis: key to the attenuated malaria vaccine? J. Infect. Dis. 191, 1573-1575

53 van Dijk, M. et al. (2005) Genetically attenuated, P36p-deficient malarial sporozoites induce protective immunity and apoptosis of infected liver cells. Proc. Natl. Acad. Sci. U.S.A. 102, 12194-12199

54 Jobe, O. et al. (2009) Immunization with radiation-attenuated Plasmodium berghei sporozoites induces liver cCD8alpha ${ }^{+} \mathrm{DC}$ that activate $\mathrm{CD} 8^{+} \mathrm{T}$ cells against liver-stage malaria. PLoS ONE 4, e5075

55 Rénia, L. et al. (2006) Do apoptotic Plasmodium-infected hepatocytes initiate protective immune responses? J. Infect. Dis. 193, 163-164

56 Sauerwein, R. et al. (2010) Empowering malaria vaccination by drug administration. Curr. Opin. Immunol. 22, 367-373

57 Accapezzato, D. et al. (2005) Chloroquine enhances human CD8 T cell responses against soluble antigens in vivo. J. Exp. Med. 202, 817-828
58 Berenzon, D. et al. (2003) Protracted protection to Plasmodium berghei malaria is linked to functionally and phenotypically heterogeneous liver memory CD8 ${ }^{+} \mathrm{T}$ cells. J. Immunol. 171, 2024-2034

59 Jobe, O. et al. (2007) Genetically attenuated Plasmodium berghei liver stages induce sterile protracted protection that is mediated by major histocompatibility complex class I-dependent interferon- $\gamma$-producing CD8 ${ }^{+}$T cells. J. Infect. Dis. 196, 599-607

60 Scheller, L. and Azad, A. (1995) Maintenance of protective immunity against malaria by persistent hepatic parasites derived from irradiated sporozoites. Proc. Natl. Acad. Sci. U.S.A. 92, 4066-4068

61 Mueller, A. et al. (2007) Genetically attenuated Plasmodium berghei liver stages persist and elicit sterile protection primarily via CD8 $\mathrm{T}$ cells. Am. J. Pathol. 171, 107-115

62 Keating, S. et al. (2005) Durable human memory T cells quantifiable by cultured enzyme-linked immunospot assays are induced by heterologous prime boost immunization and correlate with protection against malaria. J. Immunol. 175, 5675-5680

63 Todryk, S. et al. (2009) The relationship between human effector and memory T cells measured by ex vivo and cultured ELISPOT following recent and distal priming. Immunology 128, 83-91

64 Doolan, D. et al. (2003) Identification of Plasmodium falciparum antigens by antigenic analysis of genomic and proteomic data. Proc. Natl. Acad. Sci. U.S.A. 100, 9952-9957

65 Webster, D. et al. (2005) Enhanced T cell-mediated protection against malaria in human challenges by using the recombinant poxviruses FP9 and modified vaccinia virus Ankara. Proc. Natl. Acad. Sci. U.S.A. 102, $4836-4841$

66 Siegrist, C. (2004) Vaccine Immunology. In Vaccines (Plotkin, S. et al., eds), pp. 17-36, Elsevier

67 Shapiro-Shelef, M. et al. (2003) Blimp-1 is required for the formation of immunoglobulin secreting plasma cells and pre-plasma memory $\mathrm{B}$ cells. Immunity 19, 607-620

68 Hansen, S. et al. (2009) Effector memory T cell responses are associated with protection of rhesus monkeys from mucosal simian immunodeficiency virus challenge. Nat. Med. 15, 293-299

69 Querec, T. et al. (2006) Yellow fever vaccine YF-17D activates multiple dendritic cell subsets via TLR2,7,8 and 9 to stimulate polyvalent immunity. J. Exp. Med. 203, 413-424

70 Reed, S. et al. (2008) New horizons in adjuvants for vaccine development. Trends Immunol. 30, 23-32

71 Hutchings, C. et al. (2007) Combination of protein and viral vaccines induces potent cellular and humoral immune responses and enhanced protection from murine malaria challenge. Infect. Immun. 75, 58195826

72 Stewart, V. et al. (2007) Priming with an adenovirus 35circumsporozoite protein (CS) vaccine followed by RTS,S/AS01B boosting significantly improves immunogenicity to Plasmodium falciparum CS compared to that with either malaria vaccine alone. Infect. Immun. 75, 2283-2290

73 Mauduit, M. et al. (2009) A role for immune responses against non-CS components in the cross-species protection induced by immunization with irradiated malaria sporozoites. PLoS ONE 4, e7717

74 Rogers, W. et al. (1992) Characterization of Plasmodium falciparum sporozoite surface protein 2. Proc. Natl. Acad. Sci. U.S.A. 89, 91769180

75 Mazier, D. et al. (1990) Hepatic phase of malaria is the target of cellular mechanisms induced by the previous and the subsequent stages. A crucial role for liver nonparenchymal cells. Immunol. Lett. 25, 65-70

76 Frevert, U. and Nardin, E. (2008) Cellular effector mechanisms against plasmodium liver stages. Cell. Microbiol. 10, 1956-1967

77 Bonifaz, L. et al. (2004) In vivo targeting of antigens to maturing dendritic cells via the DEC-205 receptor improves T cell vaccination. J. Exp. Med. 199, 815-824

78 Wille-Reece, U. et al. (2005) Immunization with HIV-1 Gag protein conjugated to a TLR7/8 agonist results in the generation of HIV-1 Gagspecific Th1 and CD8 ${ }^{+} \mathrm{T}$ cell responses. J. Immunol. 174, 7676-7683

79 Plebanski, M. et al. (1999) Interleukin 10-mediated immunosuppression by a variant $\mathrm{CD} 4 \mathrm{~T}$ cell epitope of Plasmodium falciparum. Immunity 10 , $651-660$ 